\title{
Nonoperative Treatment Options for Patients With Sacroiliac Joint Pain
}

\author{
HEIDI PRATHER, DO, ${ }^{1}$ MICHAEL BONNETTE, MD, ${ }^{2}$ DEVYANI HUNT, MD $^{1}$ \\ ${ }^{I}$ Division of Physical Medicine and Rehabilitation, Department of Orthopaedic Surgery, Washington University School of Medicine, St. Louis, Missouri, \\ ${ }^{2}$ Department of Neurology, Washington University School of Medicine, St. Louis, Missouri
}

\begin{abstract}
Sacroiliac joint (SIJ) pain is thought to be a component of low back pain in $20 \%$ of people who suffer with it chronically. There is no consistent objective diagnostic testing that includes SIJ pain as the diagnosis and thereby it can become a diagnosis of exclusion. Treatment of SIJ pain is variable, and no set method or protocol of treatment has been found to be efficacious or reliable. Thus, the healthcare provider is often left to create an individual treatment plan based on their own experiences and expertise. The purpose of this narrative review is to describe and discuss nonoperative treatment options for patients with SIJ pain. Further, coordination of treatment options and progression of treatment will be offered.
\end{abstract}

Other \& Special Categories

Keywords: sacroiliac joint pain, low back pain, sacroiliac joint dysfunction

\section{TREATMENT OF SACROILIAC JOINT PAIN}

Patients present for treatment wanting relief of pain. This basic request cannot be lost by the healthcare provider who can be overwhelmed with the diagnosis and appropriate noninvasive treatment options for patients with sacroiliac joint (SIJ) pain. The provider must interpret various presentations and cofounding circumstances in which SIJ pain develops and then navigate a multitude of treatment options. There is no single or group of specific noninvasive treatments that is superior to another. This leaves invasive interventions to be the "go to" for confirming the diagnosis and treatment when the noninvasive approaches have failed to work. In this narrative review, we will discuss theoretical approaches to treatment based on clinical experience and available literature. We will discuss treatments for SIJ pain including pain reduction, therapeutic exercise, manual medicine, belting, and orthotics and injection procedures.

\section{Pain Reduction}

Often, SIJ pain presents as a progressive problem with fluctuations in symptoms that may be experienced with specific activities of daily living, sports, or exercise. When pain is in the acute or subacute stage, reducing inflammation may be helpful with a course of nonsteroidal anti-inflammatories schedule for 2 weeks or fewer combined with regular icing. Relative rest from these pain-provoking activities such as running, single-leg, or twisting activities can also speed the course of recovery. ${ }^{1}$ Addressing the patient's pain at the initial meeting is important and allows subsequent treatments to be better tolerated. Attention can then be directed at correcting the functional biomechanical deficit and tissue overload complex $^{2}$ through therapeutic exercise, manual medicine, belting, and orthotics as indicated.

\section{Therapeutic Exercise}

After pain has been addressed, healthcare providers often recommend a consultation and treatment recommendations from a physical therapist with the intent that a regime of therapeutic exercise will assist in correcting the functional biomechanical deficits to restore motion that also contributes to pain reduction. A home exercise program is recommended for meaningful change and hopes of prevention of recurrences of SIJ pain over time. Therapeutic exercise plays such a key role in addressing the underlying dysfunction that led to the development of pain. Unfortunately, the literature is limited on this topic, and the healthcare 
provider is left to rely on their personal training and experiences with patient success and failures.

In a randomized controlled trial of 22 people with SIJ pain comparing laser treatment to therapeutic exercise, Monticone et $\mathrm{al}^{3}$ found at 12 months, only those treated with therapeutic exercise reported a reduction in pain despite both groups no longer had positive provocative SIJ tests following either treatment. In a randomized control trial involving SIJ pain to assess the short-term benefit of therapeutic exercise as compared with manual therapy and intra-articular SIJ injection, Visser et $\mathrm{al}^{4}$ found therapeutic exercise alone to be successful in $3 / 15(20 \%)$ of patients. Multiple case reports of therapeutic exercise alone ${ }^{5-9}$ and a case series of combined therapeutic exercise and manipulation ${ }^{10}$ demonstrated decreased pain and improved function following treatment. ${ }^{5,8,10}$

Likely the reason for paucity of literature regarding SIJ pain and therapeutic exercise at this time is twofold: (1) there is great variability in the functional biomechanical deficit in people with SIJ pain; (2) the standard of care is to apply more than 1 form of treatment at a time. This makes it difficult to standardized treatment for study purposes. In general, the approach to therapeutic exercise recommendations is linked to balancing muscle length, strength, and appropriate motor control in order to absorb and transmit force across the ilium and sacrum.

A few key muscles and muscle groups require specific attention when treating SIJ pain and dysfunction. The hamstring is 1 of them and provides stability to the SIJ because of its direct attachment and/or fascial connections to the sacrotuberous ligament, an extrinsic SIJ ligament that provides joint stability. Assessing if the muscles of the pelvis are too short or too long as well as if they are too stiff will guide treatment. Muscles commonly found to be working in a suboptimal shortened position include the iliopsoas, rectus femoris, tensor fascia lata, adductors, quadratus lumborum, latissimus dorsi, and obturator internus. As muscle length and/or reduction of muscle stiffness is accomplished, strengthening of muscles that are inhibited by the biomechanical deficit can be completed. Neuromuscular reeducation and facilitation techniques are helpful with this process. Closed kinetic chain strengthening should be attempted first and then incorporated into the lumbopelvic stabilization exercises.
Similarly assessing and addressing weakness and imbalance is important. Muscles commonly found to be weak in people with SIJ pain include the gluteus medius, gluteus maximus, lower abdominals, and hamstrings. Though maintaining individual muscle flexibility and strength is important, retraining multiple muscle groups to fire in coordination is key to a successful recovery. This can be facilitated with lumbopelvic stabilization, advance proprioceptive reeducation, plyometrics, and exercise or sport-specific activities. Education regarding proper ergonomics in activities of daily living and work environment should be included. Careful attention to training techniques must also be incorporated into the program for those active in sports and exercise. Return to activities that provoke symptoms including sports and exercise should resume when a pain-free state without medications is achieved. Proper muscle balance in flexibility and strength should remain a part of the maintenance program. Careful monitoring during initial return to sport and exercises can prevent reinjury.

\section{Manual Medicine}

Manual medicine techniques are often a part of the discussion and consideration of treatment for SIJ pain. However, like any other intervention, picking the right patient with the right disorder is key to an optimal outcome. There are a number of manually trained healthcare providers from a variety of professional disciplines that offer a wide array of manual techniques that can be included within the mobilization category including high velocity low amplitude (HVLA), low velocity high amplitude, physical therapy joint mobilization, muscle energy, and active release therapy. ${ }^{1}$

In a recent systematic review of physiotherapy interventions of SIJ dysfunction, Al-Subahi et $\mathrm{al}^{11}$ found 4 studies that met the appropriate review criteria for treatment with manipulation. In a randomized control trial ${ }^{12}$ comparing manipulation (HVLA) of the SIJ alone to SIJ and lumbar manipulation, both groups reported reduced pain and disability at 1 month posttreatment. Another study ${ }^{13}$ compared mechanical force-manually assisted manipulation using a hand instrument to apply HVLA to the symptomatic SIJ. Patients in both treatment groups reported reduced pain and disability 3 weeks after treatment. Childs et $\mathrm{al}^{14}$ compared HVLA to the SIJs alone to HVLA to 
the lumbar spine and SIJs in male and female patients with lumbopelvic pain. Both groups completed home exercise programs independently. At 4day follow-up, patients in both groups reported improved pain, function, and pelvic symmetry on examination. Another combined approach study ${ }^{10}$ of exercise and manipulation, including HVLA to the SIJs, found improved pain and restoration of pelvic symmetry. The small sample size and lack of reporting of necessary repeated interventions make these data difficult to interpret for application to a large population.

In our clinical experience, if an SIJ appears to require recurrent joint mobilization, a significant muscle imbalance may still exist or the patient may have joint hypermobility locally. Some of this may be related to compliance or an inadequate neuromotor control and strength-directed program, or may be related to the patient's inherent collagen makeup that allows increase joint mobility and/or tissue laxity. Caution should be used with repetitive manipulation in the latter group. Muscle energy techniques are helpful as they require patient activation of muscle groups, and therefore pain tolerance can be more easily monitored. Special care should be taken with early treatment of the pregnant athlete, as aggressive stretching or mobilization can further aggravate symptoms secondary to pregnancy-related hypermobility. Although there is considerable focus on gaining range of motion through the lumbar spine as part of treatment, during the recovery phase, it is important to differentiate hip range of motion limits that are related to pain or soft tissue restrictions versus limitations related to bony deformity, including femoroacetabular impingement and acetabular dysplasia.

\section{Orthosis and Orthotics}

\section{SIJ Belts}

SIJ belts are used to provide compression across the ilium and thereby the SIJ with the intent to improve force closure across the joints. Subsequently, this provides proprioceptive feedback to the gluteal muscle, which can enhance their activation furthering improvement in force closure across the joints. Vleeming et $\mathrm{al}^{15}$ found that SIJ belts applied to cadaver models reduced SIJ rotation by $30 \%$. In a study assessing the influence of an SIJ belt on ligament loading via a computer model, Sichting et $\mathrm{al}^{16}$ found that the belt increased SIJ motion in the sagittal axis but decreased motion in the transverse axis. Ligament strain patterns were reduced with SIJ belt application. The ligament and SIJ motion measurements were minute. Hammer et $\mathrm{al}^{17}$ found improved physical health subscores of the shortform 36 survey, reduction in pain, and reduced rectus femoris activity with walking in 17 patients with SIJ pain using an SIJ belt as compared with 17 controls. The SIJ belt improved cadence and gait velocity in both patients with SIJ pain and controls. In this same group of patients and controls, Soisson et $\mathrm{al}^{18}$ reported minimal changes in frontal plane spinal alignment. No correlation was found with pain improvement and altered muscle activity, pelvic morphometry, or body balance in a static short-term wearing. The authors proposed that long-term effects of wearing the belt should be studied to assess for benefit in muscle activation and body balance. SIJ belts can be especially helpful in patients with SIJ hypermobility, muscle weakness or inhibition, and poor neuromotor control.

This literature does not identify responders to wearing an SIJ belt. A positive active straight leg raise found on physical examination can be a good indicator to the healthcare provider that a trial of a SIJ belt would be useful. ${ }^{19,20}$

Care must be taken to ensure that the patient is able to apply the belt appropriately. The SIJ belt should be secured posteriorly across the sacral base and anteriorly, inferior to the anterior superior iliac spines (Figure). Patients are often recommended to wear the belt during walking and standing activities. However, patients with significant pain and weakness often find the belt helpful in reducing symptoms when worn during sedentary activities as well, including at night while sleeping.

\section{Orthotics}

Other treatment considerations include orthotics and shoe modifications. A shoe lift to correct a functional leg length discrepancy can be helpful in the acute setting to manage pain with weightbearing or ambulation. However, shoe lifts should be approached with caution, as functional leg length discrepancy should be corrected with muscle rebalancing and not an orthotic. An inappropriate shoe lift can promote adaptive muscle imbalances, which may initially be asymptomatic but cause problems because of a shift in force transmission, which ultimately can change wear and tear pattern in the future. In contrast, anatomical leg length discrep- 


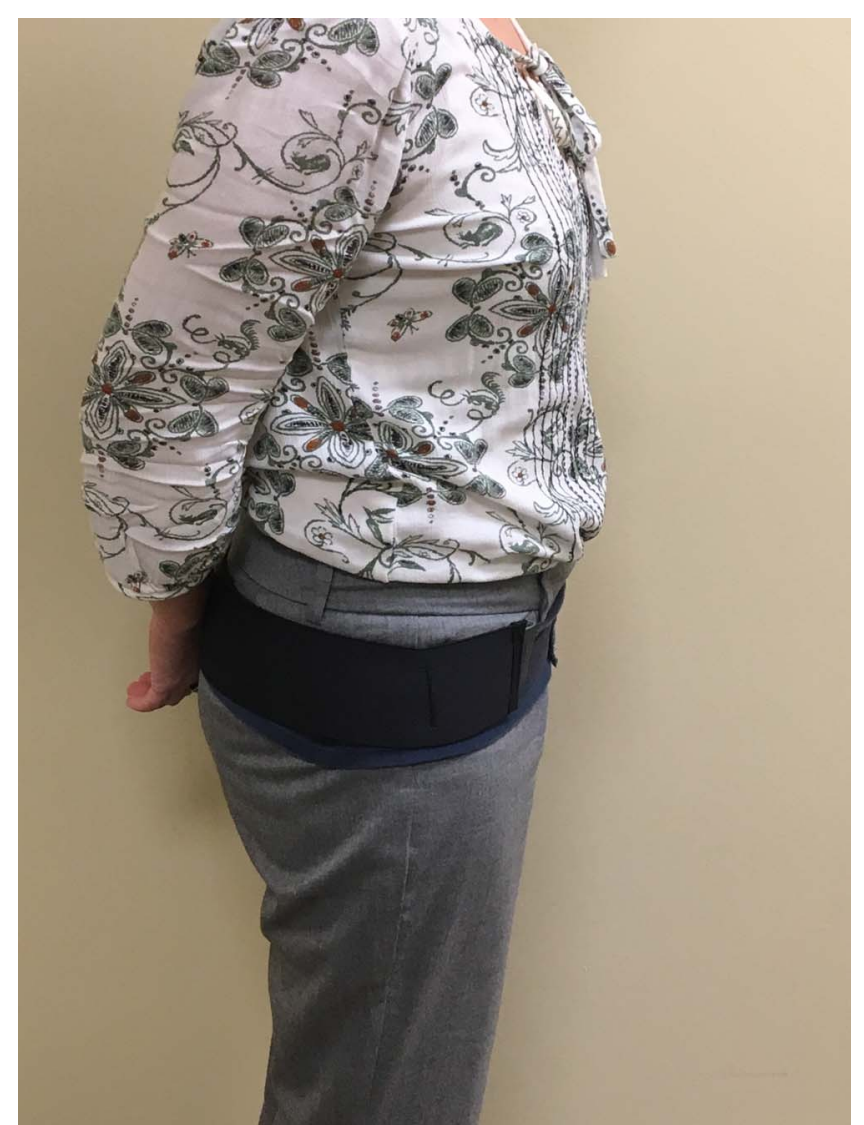

Figure. Fitting for sacroiliac joint belt.

ancies should be determined as early in treatment as possible so that the appropriate modifications can be completed.

\section{SIJ INJECTIONS AND RADIOFREQUENCY ABLATION}

Intra-articular injections and radiofrequency ablation are commonly implemented treatment options offered to patients with SIJ pain. Often these procedures will serve a dual role of both confirming the diagnosis of SIJ pain and treatment intervention. It has been well established that distending the SIJ capsule in asymptomatic patients will induce pain and injecting local anesthetic intra-articularly can reduce pain in the SIJ as well as pain radiating into the leg. ${ }^{21-23}$ The use of fluoroscopic guidance is necessary for these as intra-articular needle placement to ensure accuracy of intra-articular placement. Ultrasound and computed tomography guidance do not rule out intravascular flow and are not as effective in verifying intra-articular placement of the injectate. ${ }^{24,25}$ The capacity of the SIJ ranges from 1.0 to $2.6 \mathrm{~mL}^{21,23}$ in symptomatic patients, which argues that volumes of injectate greater than this may leak as its ventral capsule commonly has defects, thus not being specific for intra-articular SIJ pain.

Selection of patients for both intra-articular injections and radiofrequency ablation is difficult due to the complexity of diagnosis SIJ pain and distinguishing it from other relevant structures such as the sacroiliac ligaments and surrounding musculature. There is a population that benefits from these injections, but selecting for this population is difficult as neither history of symptoms nor a single or combination of examination maneuvers have been shown to be predictive of response. However, if at least 3 physical examination findings (FABER, thigh thrust, Gaenslen's, Distraction, Sacral Thrust, and Compression) are positive, there is an increase in specificity and sensitivity of intra-articular injections. $^{26}$

A review by Simopoulos et $\mathrm{al}^{27}$ showed level II to III evidence for diagnostic accuracy of sacroiliac intra-articular injections. With that said, diagnostic injections alone are not commonly performed prior to therapeutic injection with steroid as to limit the total number of injections for a patient to reduce cost, risk of infection, radiation exposure, and travel burden. $^{28}$ In a review by MacVicar et $\mathrm{al}^{28}$ to determine the appropriate use criteria for SI interventions, an intra-articular injection of local anesthetic and steroid was an appropriate first intervention in patients with SIJ region pain for greater than 1 month, at an intensity of $4 / 10$, and caused functional limitation regardless of whether or not conservative treatment had been provided. ${ }^{28}$ Additionally, Schneider et $\mathrm{al}^{29}$ found that despite the expected bimodal pain relief response to an injection of local anesthetic and steroid, patients, who do not achieve immediate relief after the procedure, are unlikely to have substantial relief at 2- or 4-week follow-up.

The evidence for intra-articular steroid injections has been given moderate $\operatorname{grade}^{26}$ and level IV, ${ }^{27}$ which is not overwhelming but understandable due to the aforementioned difficulty in patient selection and differentiating the SIJ as the primary source of pain in the low back pain population.

The SIJ is thought to have both ventral and dorsal innervation, whereas the sacroiliac ligaments have innervation from the lateral branches of the sacral dorsal rami as well. ${ }^{2,30}$ Radiofrequency ablation of these lateral branches is performed to 
treat SIJ pain but likely gives pain relief to not only intra-articular mediated pain but also the entire sacroiliac complex. A review by Simopoulos et $\mathrm{al}^{27}$ in 2015 found level III evidence for the use of cooled radiofrequency ablation and level $\mathrm{V}$ evidence for the use of conventional radiofrequency ablation. The use of radiofrequency ablation has been suggested to give longer duration of symptom relief in some patients than intra-articular steroid injections in a 2018 study. $^{31}$

Interestingly, intra-articular blocks are still commonly used as a diagnostic screening test prior to radiofrequency ablation despite the evidence of dorsal and ventral innervation. ${ }^{21}$ Current practice recommendations for lumbar branch radiofrequency ablation include 2 factors for patient selection: 2to $3-$ month minimum duration of pain and greater than $50 \%$ pain relief from diagnostic injection. Additionally, repeat ablation procedures were deemed inappropriate if the patient did not receive at least $50 \%$ pain relief or the duration of relief was less than 3 months after the first procedure. ${ }^{28}$ Several studies, including a cadaveric study by Roberts et $a{ }^{32},{ }^{32}$ found that many of the commonly used ablation techniques fail to capture all of the lateral branches and suggested that further study into location of ablation sites and ablation techniques as well as valid diagnostic blocks and rigorous patient selection criteria are crucial in optimizing outcomes.

In summary, the accepted method of treatment of patients with SIJ pain includes a trial of multiple methods of treatment that should be guided by the patient's history and clinical symptom complex. Caution should be taken to not to continue to mobilize the joints of patients with increase joint mobility or connective tissue laxity. Procedures including injections and radiofrequency denervation of the SIJ can be useful tools to reduce pain when noninvasive treatments are unsuccessful.

\section{REFERENCES}

1. Prather H, Hunt D. Conservative management of low back pain, part I. Sacroiliac joint pain. Dis Mon. 2004;50(12):670-683.

2. Herring SA. Rehabilitation of muscle injuries. Med Sci Sports Exerc. 1990;22(4):453-456.

3. Monticone M, Barbarino A, Testi C, Arzano S, Moschi A, Negrini S. Symptomatic efficacy of stabilizing treatment versus laser therapy for sub-acute low back pain with positive tests for sacroiliac dysfunction: a randomised clinical controlled trial with 1 year follow-up. Eura Medicophys. 2004;40(4):263268.
4. Visser LH, Woudenberg NP, de Bont J, et al. Treatment of the sacroiliac joint in patients with leg pain: a randomizedcontrolled trial. Eur Spine J. 2013;22(10):2310-2317.

5. Boyle KL. Managing a female patient with left low back pain and sacroiliac joint pain with therapeutic exercise: a case report. Physiother Can. 2011;63(2):154-163.

6. Cho BY, Yoon JG. The effect of gait training with shoe inserts on the improvement of pain and gait in sacroiliac joint patients. J Phys Ther Sci. 2015;27(8):2469-2471.

7. Yoo WG. Effects of double air-cushion biofeedback exercises in a patient with sacroiliac joint pain. J Phys Ther Sci. 2015;27(11):3605-3606.

8. Yoo WG. Effects of individual strengthening exercises for the stabilization muscles on the nutation torque of the sacroiliac joint in a sedentary worker with nonspecific sacroiliac joint pain. J Phys Ther Sci. 2015;27(1):313-314.

9. Yoo WG. Effect of the single-leg, lateral oblique, decline squat exercise on sacroiliac joint pain with knee pain. $J$ Phys Ther Sci. 2016;28(9):2688-2689.

10. Barbosa AC, Martins FL, Barbosa MC, Dos Santos RT. Manipulation and selective exercises decrease pelvic anteversion and low-back pain: a pilot study. J Back Musculoskelet Rehabil. 2013;26(1):33-36.

11. Al-Subahi M, Alayat M, Alshehri MA, et al. The effectiveness of physiotherapy interventions for sacroiliac joint dysfunction: a systematic review. J Phys Ther Sci. 2017;29(9):1689-1694.

12. Kamali F, Shokri E. The effect of two manipulative therapy techniques and their outcome in patients with sacroiliac joint syndrome. J Bodyw Mov Ther. 2012;16(1):29-35.

13. Shearar KA, Colloca CJ, White HL. A randomized clinical trial of manual versus mechanical force manipulation in the treatment of sacroiliac joint syndrome. $J$ Manipulative Physiol Ther. 2005;28(7):493-501.

14. Childs JD, Piva SR, Erhard RE. Immediate improvements in side-to-side weight bearing and iliac crest symmetry after manipulation in patients with low back pain. $J$ Manipulative Physiol Ther. 2004;27(5):306-313.

15. Vleeming A, Buyruk HM, Stoeckart R, Karamursel S, Snijders CJ. An integrated therapy for peripartum pelvic instability: a study of the biomechanical effects of pelvic belts. Am J Obstet Gynecol. 1992;166(4):1243-1247.

16. Sichting F, Rossol J, Soisson O, Klima S, Milani T, Hammer N. Pelvic belt effects on sacroiliac joint ligaments: a computational approach to understand therapeutic effects of pelvic belts. Pain Physician. 2014;17(1):43-51.

17. Hammer N, Mobius R, Schleifenbaum S, et al. Pelvic belt effects on health outcomes and functional parameters of patients with sacroiliac joint pain. PLoS One. 2015;10(8):e0136375.

18. Soisson O, Lube J, Germano A, et al. Pelvic belt effects on pelvic morphometry, muscle activity and body balance in patients with sacroiliac joint dysfunction. PLoS One. 2015;10(3):e0116739.

19. Mens JM, Vleeming A, Snijders CJ, Stam HJ, Ginai AZ. The active straight leg raising test and mobility of the pelvic joints. Eur Spine J. 1999;8(6):468-473.

20. O'Sullivan PB, Beales DJ, Beetham JA, et al. Altered motor control strategies in subjects with sacroiliac joint pain during the active straight-leg-raise test. Spine (Phila Pa 1976). 2002;27(1):E1-E8.

21. Dreyfuss P, Henning T, Malladi N, Goldstein B, Bogduk 
N. The ability of multi-site, multi-depth sacral lateral branch blocks to anesthetize the sacroiliac joint complex. Pain Med. 2009;10(4):679-688.

22. Fortin JD, Aprill CN, Ponthieux B, Pier J. Sacroiliac joint: pain referral maps upon applying a new injection/ arthrography technique. Part II: Clinical evaluation. Spine (Phila Pa 1976). 1994;19(13):1483-1489.

23. Fortin JD, Tolchin RB. Sacroiliac arthrograms and postarthrography computerized tomography. Pain Physician. 2003;6(3):287-290.

24. Hansen HC. Is fluoroscopy necessary for sacroiliac joint injections? Pain Physician. 2003;6(2):155-158.

25. Rosenberg JM, Quint TJ, de Rosayro AM. Computerized tomographic localization of clinically-guided sacroiliac joint injections. Clin J Pain. 2000;16(1):18-21.

26. Kennedy DJ, Engel A, Kreiner DS, Nampiaparampil D, Duszynski B, MacVicar J. Fluoroscopically guided diagnostic and therapeutic intra-articular sacroiliac joint injections: a systematic review. Pain Med. 2015;16(8):1500-1518.

27. Simopoulos TT, Manchikanti L, Gupta S, et al. Systematic review of the diagnostic accuracy and therapeutic effectiveness of sacroiliac joint interventions. Pain Physician. 2015; 18(5):E713-E756.

28. MacVicar J, Kreiner DS, Duszynski B, Kennedy DJ. Appropriate use criteria for fluoroscopically guided diagnostic and therapeutic sacroiliac interventions: results from the spine intervention society convened multispecialty collaborative. Pain Med. 2017;18(11):2081-2095.

29. Schneider BJ, Huynh L, Levin J, Rinkaekan P, Kordi R, Kennedy DJ. Does immediate pain relief after an injection into the sacroiliac joint with anesthetic and corticosteroid predict subsequent pain relief? Pain Med. 2018;19(2):244-251.

30. MacVicar J, King W, Landers MH, Bogduk N. The effectiveness of lumbar transforaminal injection of steroids: a comprehensive review with systematic analysis of the published data. Pain Med. 2013;14(1):14-28.

31. Dutta K, Dey S, Bhattacharyya P, Agarwal S, Dev P. Comparison of efficacy of lateral branch pulsed radiofrequency denervation and intraarticular depot methylprednisolone injection for sacroiliac joint pain. Pain Physician. 2018;21(5):489496.

32. Roberts SL, Stout A, Loh EY, Swain N, Dreyfuss P, Agur AM. Anatomical comparison of radiofrequency ablation techniques for sacroiliac joint pain. Pain Med. 2018;19(10):1924 1943.

Disclosures and COI: All authors are employees of Washington University School of Medicine and have no conflicts of interest in this article and have no financial disclosures.

Corresponding Author: Heidi Prather, DO, Professor, Department of Orthopaedic Surgery, Washington University School of Medicine, St. Louis, Missouri, 14532 South Outer Forty Rd, Chesterfield, MO 63017. Phone: (314) 514-3514; Fax: (314) 514-3555; Email: pratherh@wustl.edu.

Published 10 February 2020

This manuscript is generously published free of charge by ISASS, the International Society for the Advancement of Spine Surgery. Copyright (c) 2020 ISASS. To see more or order reprints or permissions, see http://ijssurgery.com. 